Check for updates

Cite this: RSC Adv., 2020, 10, 43242

Received 1st October 2020

Accepted 18th November 2020

DOI: 10.1039/d0ra08390a

rsc.li/rsc-advances

\section{Synthesis and charge transfer characteristics of a ruthenium-acetylide complex $\dagger$}

\author{
Robert Kuhrt, (DD *a Po-Yuen Ho, ${ }^{\text {b }}$ Martin Hantusch, ${ }^{a}$ Franziska Lissel, ${ }^{\text {b }}$ \\ Olivier Blacque, (D) Martin Knupfer ${ }^{\mathrm{a}}$ and Bernd Büchner ${ }^{\mathrm{a}}$
}

\begin{abstract}
A novel ruthenium-acetylide complex was synthesised and characterised in solid state and solution. Thin films of the complex were evaporated on silver and gold foils in ultra high vacuum in order to probe the electronic properties with photoemission spectroscopy. The charge transfer characteristics of the complex with the strong acceptor $\mathrm{F}_{6} \mathrm{TCNNQ}$ were investigated by UV-vis absorption in solution as well as at an interface with photoemission spectroscopy. A new excitation in the former optical gap of the pristine materials was probed in solution. Moreover, it was possible to identify the oxidised complex as well as the reduced acceptor by X-ray photoemission spectroscopy. In particular, our data reveal that oxidation of the complex mainly occurs at the Ru centre. The charge transfer can be characterised as localised and mainly ionic although signs of a reaction of the acceptors aminogroups with the ruthenium-acetylide complex were found.
\end{abstract}

\section{Introduction}

Ruthenium-acetylide complexes were initially developed for the field of base-metal catalysis. More recently, their charge transfer behaviour was investigated by various methods, including mechanically controllable break junction (MCBJ), where this class of complexes demonstrated high conductivity ${ }^{1,2}$ and spectro-electrochemical analysis, in which their redox responsiveness and different charge transfer modes such as metal-toligand charge-transfer (MLCT), ligand-to-metal charge-transfer (LMCT) and even inter valence charge transfer (IVCT) were studied. ${ }^{3-5}$ Yet while the through-bond charge transfer behaviours were investigated by the above-mentioned methods, their through-space (or inter-molecular) charge transfer ability still remains ambiguous despite of its importance for organic electronic applications. Furthermore, unlike other Ru-compounds, which have been used as dopants for (organic) semiconductors, the doping ability of ruthenium-acetylide complexes is still unclear. In this study, we seek to fill this gap, and study the inter-molecular charge transfer ability of ruthenium-acetylide complexes. An exemplary complex, trans-[Ru(dppe $\left.)_{2}(\mathrm{~T})_{2}\right]$, was synthesized, which can be understood as the repeating unit of a redox-active semiconducting polymetallayne. ${ }^{6}$ The acetylenes

${ }^{a}$ Leibniz-Institut für Festkörper- und Werkstoffforschung Dresden, Helmholtzstr. 20, 01069 Dresden, Germany. E-mail: r.kuhrt@ifw-dresden.de

${ }^{b}$ Leibniz-Institut für Polymerforschung Dresden, Hohe Str. 6, 01069 Dresden, Germany 'Department of Chemistry, University of Zurich, Winterthurerstrasse 190, 8057 Zürich, Switzerland

$\dagger$ Electronic supplementary information (ESI) available. CCDC 2010145. For ESI and crystallographic data in CIF or other electronic format see DOI: 10.1039/d0ra08390a are end-capped with thiophene in order to yield a stable acetylide structure. The electron-rich character of the ruthenium moiety is utilized ${ }^{7}$ and charge transfer to an electron acceptor is observed and studied by photoemission spectroscopy. A charge transfer interface was formed with the strong electron acceptor $\mathrm{F}_{6}$ TCNNQ, which was studied recently in combination with various other organic semiconductors. ${ }^{8,9}$

\section{Synthesis and characterisation}

trans- $\left[\mathrm{Ru}(\mathrm{dppe})_{2}(\mathrm{~T})_{2}\right]$ was synthesized via a dehydrohalogenation reaction starting from cis- $\mathrm{Ru}(\mathrm{dppe})_{2} \mathrm{Cl}_{2}$ (Fig. 1). cis$\mathrm{Ru}$ (dppe $)_{2} \mathrm{Cl}_{2}(0.50 \mathrm{~g}, 0.52 \mathrm{mmol})$ was added into a $100 \mathrm{~mL}$ round-bottom flask with $50 \mathrm{~mL}$ of dichloromethane (DCM). Sodium hexafluorophosphate $(0.29 \mathrm{~g}, 1.7 \mathrm{mmol})$ was then added into the same flask. Once the hexafluorophosphate was added, the colour of the solution changed from bright yellow to deep red immediately. 2-Ethynylthiophene $(0.18 \mathrm{~g}, 1.62 \mathrm{mmol})$ and trimethylamine $(0.32 \mathrm{~g}, 3.13 \mathrm{mmol})$ were then added into the mixture. The colour of the mixture turned from red to yellow. The reaction mixture was stirred at room temperature overnight, then acetonitrile (ACN) was added and the DCM was
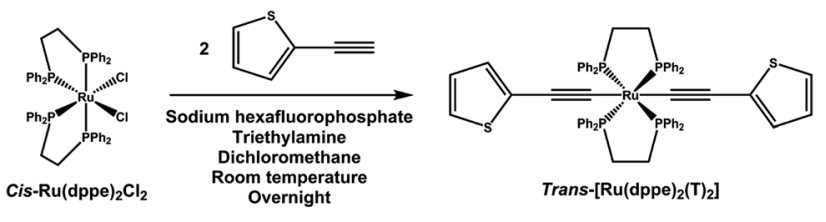

$85 \%$

Fig. 1 Synthetic route to trans-[Ru(dppe $\left.)_{2}(T)_{2}\right]$. 
removed on rotary evaporator causing a precipitate to form. The resulting precipitate was washed with ACN and then with hexane. The target compound was afforded as a yellow powder (0.49 g, $0.44 \mathrm{mmol}$, yield $=84.9 \%)$. Crystals suitable for X-ray analysis were grown from DCM.

The compound was fully characterized using ${ }^{1} \mathrm{H},{ }^{13} \mathrm{C}$ and ${ }^{31} \mathrm{P}$ NMR, UV-vis in solution and thin film, Raman, electrospray ionization mass spectrometry (ESI-MS), elemental analysis and cyclic voltammetry. Furthermore the structure was confirmed by a single crystal X-ray diffraction experiment. Detailed information on the experimental results can be found in the ESI, $\uparrow$ including the corresponding figures.

As expected for a centrosymmetric diamagnetic compound, the ${ }^{3} 1 \mathrm{P}$ NMR spectra shows a singlet at $52.9 \mathrm{ppm}$, corresponding to the four chemically equivalent phosphorous atoms of the bidentate ligands. The symmetric vibration of the acetylide is detected in the Raman spectrum at $2064 \mathrm{~cm}^{-1}$. The cyclic voltammogram exhibits one fully reversible oxidation event at $-0.073 \mathrm{~V} v s$. the ferrocene/ferrocenium redox pair. The oxidation is metal-centred and corresponds to the $\mathrm{Ru}(\mathrm{II}) / \mathrm{Ru}$ (III) transition.

The single crystal X-ray diffraction data were collected at 160 K on a Rigaku Oxford Diffraction Synergy (Pilatus 200K detector) diffractometer equipped with an Oxford liquid-nitrogen Cryostream cooler and using the $\mathrm{Cu} K \alpha$ radiation $(\gamma=1.54184 \AA)$. The pre-experiment, data collection, data reduction and analytical absorption correction ${ }^{\mathbf{1 0}}$ were carried out with the program suite CrysAlisPro (version 1.171.40.39a). ${ }^{11}$ Using Olex $2,{ }^{12}$ the structure was solved with the SHELXT ${ }^{13}$ small molecule structure solution program and refined with the SHELXL program package ${ }^{\mathbf{1 4}}$ by fullmatrix least-squares minimization on F2. In the crystal structure (Fig. 2), the ruthenium centre lies on a centre of inversion, only half of the molecule was refined, the second part is reproduced by a symmetry operation. The thiophene ligand is disordered over two sets of positions with site-occupancy factors of $0.392(3)$ and $0.608(3)$. The hydrogen atoms were placed in calculated positions by means of a riding model with $\mathrm{C}-\mathrm{H}=0.95-0.99 \AA$ and $U k_{\text {iso }}=1.2 U k_{\text {eq }}(\mathrm{C})$. The crystal data parameters are summarized in Table S1. $\dagger$

\section{Experimental details}

The UV-vis absorption measurements were carried out on a PerkinElmer Lambda800 instrument on an approx. $230 \mathrm{~nm}$

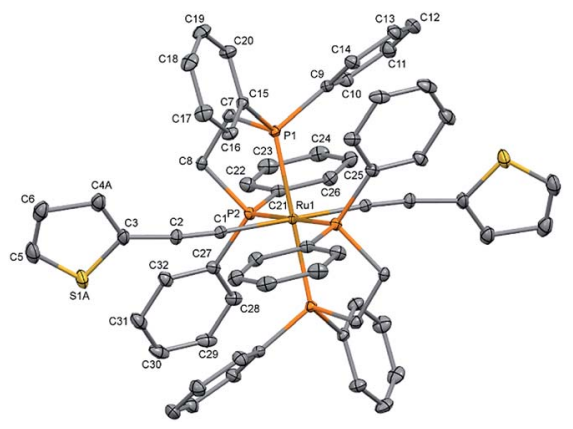

Fig. 2 Molecular structure of trans-[Ru(dppe $\left.)_{2}(T)_{2}\right]$ (all hydrogen atoms are omitted for clarity). The thermal ellipsoids are drawn at the $20 \%$ probability level. thick film prepared from a $20 \mathrm{mg} \mathrm{mL}^{-1}$ trans $-\left[\mathrm{Ru}(\mathrm{dppe})_{2}(\mathrm{~T})_{2}\right]$ chloroform solution. The glass substrate was sonicated in acetone and isopropanol bath for 10 minutes each. The solution was spin-coated on the glass substrate with a spin-rate of $500 \mathrm{rpm}$ and an acceleration of $100 \mathrm{rpm} \mathrm{s}^{-1}$ for $60 \mathrm{~s}$.

The thin films for photoemission spectroscopy were deposited on sputter cleaned gold and silver foils by powder sublimation in ultra high vacuum conditions ( $p=\approx 10^{-9}$ mbar) with a growth rate of approximately $0.3 \mathrm{~nm} \min ^{-1}$, which was monitored by a quartz crystal micro balance. The strong acceptor $\mathrm{F}_{6} \mathrm{TCNNQ}$, purchased from Novaled $\mathrm{GmbH}$ was used to form a charge transfer interface. The evaporation temperatures were approx. $270{ }^{\circ} \mathrm{C}$ for trans-[Ru(dppe $\left.)_{2}(\mathrm{~T})_{2}\right]$ and $245{ }^{\circ} \mathrm{C}$ for $\mathrm{F}_{6}$ TCNNQ. A monochromated Al-K $\alpha$ X-ray source $(h \nu=1486.7$ $\mathrm{eV}$ ) was used for X-ray photoemission spectroscopy (XPS) and a non-monochromated UVS-300 $(h \nu=21.21 \mathrm{eV}) \mathrm{He}$ gasdischarge lamp for UV photoemission spectroscopy (UPS). The measurements were carried out with a SPECS PHOIBOS-150 analyser operating with pass energies of $3 \mathrm{eV}$ for UPS and $10 \mathrm{eV}$ for XPS. The XPS core level spectra were calibrated using the $\mathrm{Au} 4 \mathrm{f}_{7 / 2}$ and $\mathrm{Ag} 3 \mathrm{~d}_{5 / 2}$ core level emission at a binding energy of $84.0 \mathrm{eV}$ and $368.3 \mathrm{eV}$, and the UPS valence spectra are referred to the Fermi-cutoff of the metal substrates at $0.0 \mathrm{eV}$. Furthermore, the valence spectra were corrected for contributions of $\mathrm{He} 1_{\beta}$ and $\mathrm{He} 1_{\gamma}$ radiation. It was assumed that the spectra resulting from these satellites have the same shape with intensities of $1.8 \%\left(\mathrm{He} 1_{\beta}\right)$ and $0.5 \%\left(\mathrm{He} 1_{\gamma}\right)$ of the $\mathrm{He} 1_{\alpha}$ signals and are shifted by $1.87 \mathrm{eV}\left(\mathrm{He} 1_{\beta}\right)$ and $2.52 \mathrm{eV}\left(\mathrm{He} 1_{\gamma}\right)$. To obtain the correct high binding energy cutoff (HBEC), a bias voltage of $5 \mathrm{~V}$ was applied to the sample.

In addition to the quartz micro balance, the film thickness was estimated by the intensity variation of the substrate metal core level peak and applying the method of Seah and Dench, ${ }^{15}$ assuming a homogeneous film growth. The spectra presented in this study are referred to the ionisation energy $\left(E_{\mathrm{I}}\right)$ which is given by:

$$
E_{\mathrm{I}}=E_{\mathrm{B}}+\phi
$$

with the binding energy $E_{\mathrm{B}}$ and the work function

$$
\phi=21.21 \mathrm{eV}-\mathrm{HBEC}
$$

For the charge transfer interface measurements, the bottom layer with a thickness of $3 \mathrm{~nm}$ to $5 \mathrm{~nm}$ is deposited first, followed by the stepwise deposition of the top layers. Both deposition sequences, with the acceptor $\mathrm{F}_{6}$ TCNNQ as bottom layer and with trans-[Ru(dppe $\left.)_{2}(\mathrm{~T})_{2}\right]$ as bottom layer were prepared. In order to probe changes in the electronic configuration, the core levels (C 1s, P 2p, S 2p, Ru 3d, N 1s, F 1s and Au 4f) as well as the valence region and the HBEC are measured after each deposition step.

\section{Spectroscopic analysis}

\subsection{Properties of thin films}

The electronic and optical properties of trans-[Ru(dppe $\left.)_{2}(\mathrm{~T})_{2}\right]$ were studied in various ways. In the following, trans$\left[\mathrm{Ru}(\mathrm{dppe})_{2}(\mathrm{~T})_{2}\right]$ will be referred to as ruthenium complex (RuC). 


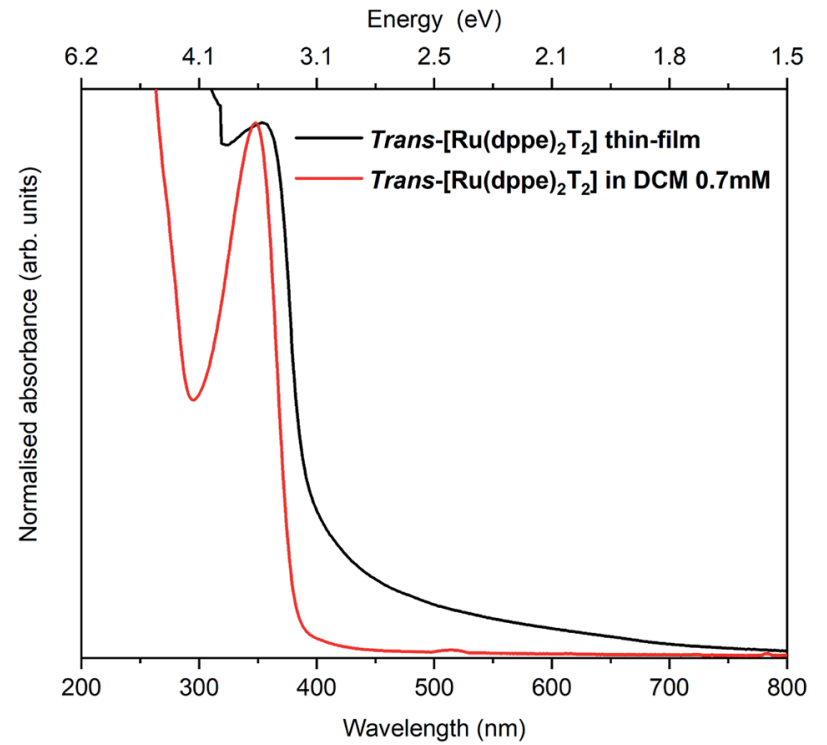

Fig. 3 Absorption spectra of a spin coated RuC film on a glass substrate (black) and a DCM solution of $0.7 \mathrm{mmol} \mathrm{L}^{-1} \mathrm{RuC}$.

First, the optical absorption was measured in solution and solid form. The solution was prepared by dissolving the complex in dichloromethane with a concentration of $0.7 \mathrm{mmol} \mathrm{L}^{-1}$ and the film was prepared by spin-coating. Fig. 3 shows the absorption spectra of film and solution. The optical gap is determined as $3.1 \mathrm{eV}$ for the film and $3.2 \mathrm{eV}$ for the solution. The very similar values indicate that the intermolecular interactions in the film do not significantly differ from those of the molecules in solution.

For the photoemission study, a film of approx. $7.5 \mathrm{~nm}$ thickness was evaporated onto a silver foil. The XP spectra of the core levels as well as the UP spectra of the valence region are displayed in Fig. 4a shows the $\mathrm{C} 1 \mathrm{~s}$ and $\mathrm{Ru} 3 \mathrm{~d}$ region. It consists of one strong broad peak at an ionisation energy $\left(E_{\mathrm{i}}\right)$ of about $288.8 \mathrm{eV}$ with a smaller peak at $287.0 \mathrm{eV}$. The $\mathrm{Ru} 3 \mathrm{~d}_{5 / 2}$ feature is detected at $284.7 \mathrm{eV}$ with the $3 \mathrm{~d}_{3 / 2}$ line superimposed by the broad $\mathrm{C}$ 1s peak. Fig. 4(b) shows the same spectrum after subtracting the $\mathrm{Ru} 3 \mathrm{~d}$ features. The molecule contains 64 carbon atoms from which 56 are $\mathrm{sp}^{2}$ hybridised (double bonds) and 4 each $\mathrm{sp}$ (triple bonds) and $\mathrm{sp}^{3}$ hybridised (single bonds) giving a ratio of $14: 1: 1$. We assign the feature at around $287 \mathrm{eV}$ to the sp hybridised carbon atoms because the two electrons forming $\pi$-bonds provide additional screening of the $\mathrm{C} 1 \mathrm{~s}$ photohole resulting in a lower binding energy and therefore a lower $E_{\mathrm{i}}$. Consequently the C 1s line of the $\mathrm{sp}^{3}$ hybridised carbons must lie at a higher $E_{\mathrm{i}}$ and the $\mathrm{sp}^{2}$ hybridised in between. The ratio of $14: 1: 1$ could be reproduced by the area ratios of the fits presented in Fig. $4 \mathrm{~b}$.

The emission from S $2 p$ and P 2 p core levels (Fig. $4 \mathrm{~b}$ and c) results in a broadened spin-orbit feature which is in agreement with the molecular structure and the equivalent positions of the $\mathrm{S}$ and $\mathrm{P}$ atoms. We assign the broadening to disorder in the films with respect to the molecular arrangement (Fig. $4 \mathrm{~b}$ and c).

A comparison of the peak areas of the $\mathrm{Ru} 2 \mathrm{p}$ doublet and the C 1s peaks weighted with the atomic sensitivity factors and the number of respective atoms per molecule showed the same

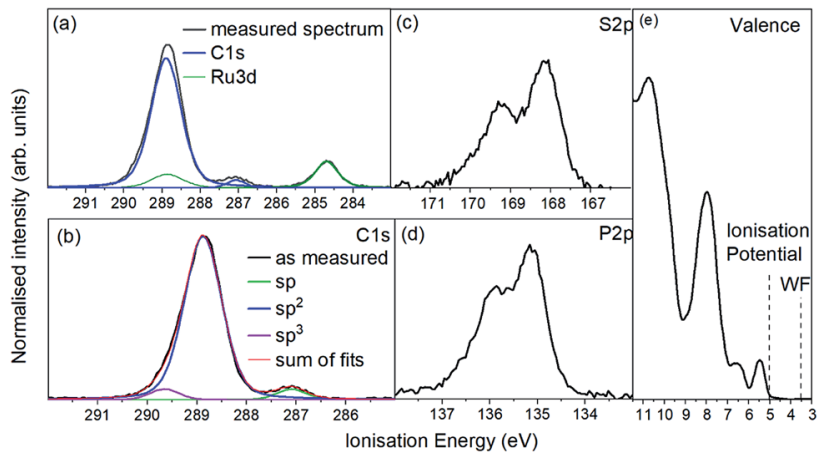

Fig. 4 Photoemission spectra of a $7.0 \mathrm{~nm}$ RuC film on a silver foil. (a) C 1s (blue) and Ru 3d (green), (b) only C 1s line after subtraction of Ru 3d doublet, (c) S 2p, (d) P 2p and (d) UP valence spectrum.

stoichiometric composition in the film as in the molecule, indicating that the molecules stayed intact during evaporation. Additionally, an IR spectrum of a film was compared to a powder spectrum which showed good agreement (ESI $\dagger$ ).

The UP spectrum of the valence region (Fig. 4d) shows the lowest ionisation energy feature of the film, representing the HOMO, with a spectral onset at $5.0 \mathrm{eV}$. This value was also found in cyclic voltammetry, which again confirms the similar intermolecular interaction in film and solution. The work function of the film, determined by the position of the HBEC, was $3.5 \mathrm{eV}$. Together with the optical gap of around $3 \mathrm{eV}$ it can be concluded that the RuC is an organic intrinsic semiconductor with the Fermi energy in the middle of the HOMOLUMO gap. An energy level diagram is shown in Fig. 5.

In order to study the charge transfer properties of the molecule, the strong electron acceptor hexafluorotetracyanonaphthoquinodimethane $\left(\mathrm{F}_{6} \mathrm{TCNNQ}^{\mathbf{1 6}}\right.$ was

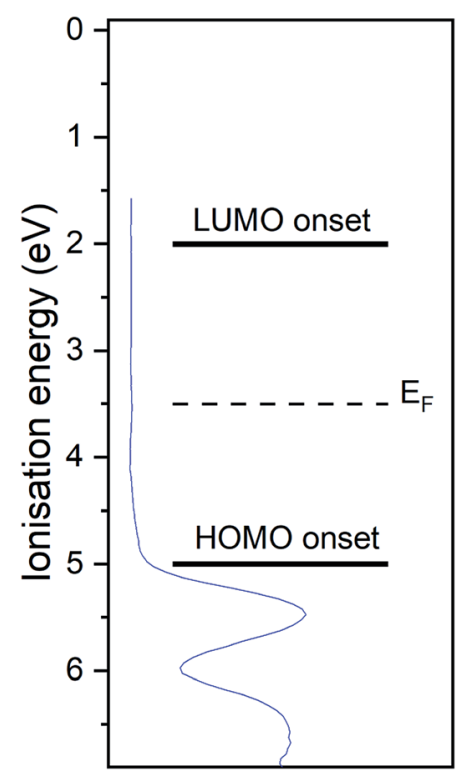

Fig. 5 Energy level diagram of RuC. Values are based on photoemission and UV-vis spectra. Valence UP spectrum of $7.0 \mathrm{~nm}$ RuC on silver foil is shown in blue. 


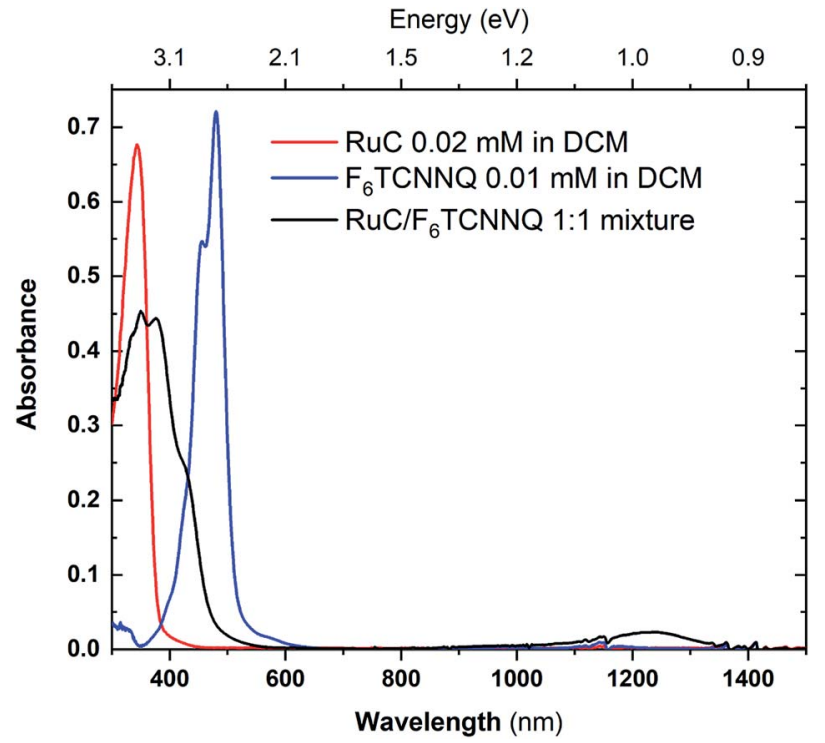

Fig. 6 UV-vis absorption spectra of pristine materials RuC (red) and $\mathrm{F}_{6} \mathrm{TCNNQ}$ (blue) and the 1:1 mixture (black) solved in DCM.

used. Its reported electron affinity of $5.6 \mathrm{eV}$ (ref. 17) is larger than the determined ionisation potential of $5.0 \mathrm{eV}$ of the RuC, which should energetically enable a charge transfer from the RuC to $\mathrm{F}_{6} \mathrm{TCNNQ}$. In the next section, the charge transfer reaction is analysed spectroscopically, first optically in a mixed solution and than at an interface with photoemission spectroscopy.

\subsection{Charge transfer with acceptor $F_{6} T C N N Q$}

In order to investigate a possible charge transfer reaction with the acceptor $\mathrm{F}_{6}$ TCNNQ, both materials were mixed in a $1: 1$ ratio in solution. The UV-vis absorption spectra of the mixture as well as the pristine materials are shown in Fig. 6. The absorption spectrum of pristine $\mathrm{F}_{6}$ TCNNQ is characterised by an excitation onset at around $500 \mathrm{~nm}$ which corresponds to an optical gap of around $2.4 \mathrm{eV}$ which is in accordance to published values. ${ }^{16}$ The RuC optical absorption spectrum shows a strong peak with a spectral onset around $400 \mathrm{~nm}$ or $3.1 \mathrm{eV}$. Upon mixing of both materials a new broad feature below the optical gap of the pristine compounds appears at around $1250 \mathrm{~nm}$. This peak is assigned to intermolecular charge transfer from the RuC to $\mathrm{F}_{6}$ TCNNQ. Previous studies of molecular semiconductors doped with $\mathrm{F}_{6}$ TCNNQ in solid and dissolved form have identified the absorption spectrum of reduced $\mathrm{F}_{6}$ TCNNQ. ${ }^{18-20}$ According to those publications the spectrum of $\mathrm{F}_{6} \mathrm{TCNNQ}^{-}$consists of a peak at $1140 \mathrm{~nm}$ which is followed by vibronic satellites at smaller wavelengths. The excitation energy in our case is smaller (around $960 \mathrm{~nm}$ ) and we cannot resolve satellites. This indicates that no pure reduced $\mathrm{F}_{6}$ TCNNQ is formed and instead some reaction between the two compounds is occurring. To further elucidate the charge transfer reaction, an interface of both material is investigated by photoemission spectroscopy.

The photoemission results of the charge transfer interface are displayed in the following manner: the sequence with the acceptor $\mathrm{F}_{6} \mathrm{TCNNQ}$ as bottom layer is shown on the left and the reverse direction on the right. The spectra are normalized and stacked in dependence of the film thickness in order to compare peak position and shape.

The XPS spectra of the Ru $3 d_{5 / 2}$ core level spectra are shown in Fig. 7. The Ru $3 d_{3 / 2}$ peak at $290.7 \mathrm{eV}$ (ref. 21) is superimposed by the large $\mathrm{C} 1 \mathrm{~s}$ peak that starts at an $E_{\mathrm{i}}$ of about $287 \mathrm{eV}$. The bottom curve on the right shows the $\mathrm{Ru} 3 \mathrm{~d}_{5 / 2}$ feature of a pristine RuC film of $5.0 \mathrm{~nm}$ thickness that can be fitted by a Voigt profile with a predominantly Gaussian contribution. Deposition of a thin layer of $\mathrm{F}_{6}$ TCNNQ (spectrum above) causes a shift of about $0.6 \mathrm{eV}$ to higher $E_{\mathrm{i}}$. This is a first indication of a charge transfer from the RuC to $\mathrm{F}_{6}$ TCNNQ since an accumulation of charge in the acceptor layer causes a dipolar layer at the interface which increases the energy required to emit a $\mathrm{Ru} 3 \mathrm{~d}$ core level electron. The opposite energetic shift is observed for the opposite deposition sequence (left).

Apart from the energy shifts, changes in the spectral shape are observed. When evaporating a thin layer of RuC on $\mathrm{F}_{6}$ TCNNQ (bottom left) a shoulder at higher $E_{\mathrm{i}}$ appears. Considering a charge transfer from the RuC to $\mathrm{F}_{6}$ TCNNQ, the absence of a valence electron results in diminished screening of the photohole which leads to a higher ionisation energy for the core level electrons. Therefore the additional feature can be attributed to an oxidised Ru centre of the molecules. For higher coverages, the contribution of the RuC cation to the overall spectrum decreases because additional RuC molecules far from the interface region are not oxidised. The topmost spectrum was taken after tempering the sample with a $5.0 \mathrm{~nm}$ RuC coverage at $100{ }^{\circ} \mathrm{C}$ for $1 \mathrm{~h}$. This lead to a mixing of the molecules by diffusion, resulting in a stronger cation signal. The opposite deposition sequence with the RuC as bottom layer and a stepwise deposition of $\mathrm{F}_{6}$ TCNNQ is shown on the right. Here, the share of the cation signal is increasing for increasing $\mathrm{F}_{6}$ TCNNQ coverage. This can be explained by the information depth of the photoelectron spectroscopy technique. With low $\mathrm{F}_{6}$ TCNNQ coverage, the $\mathrm{Ru} 3 \mathrm{~d}$ signal mainly stems from unreacted

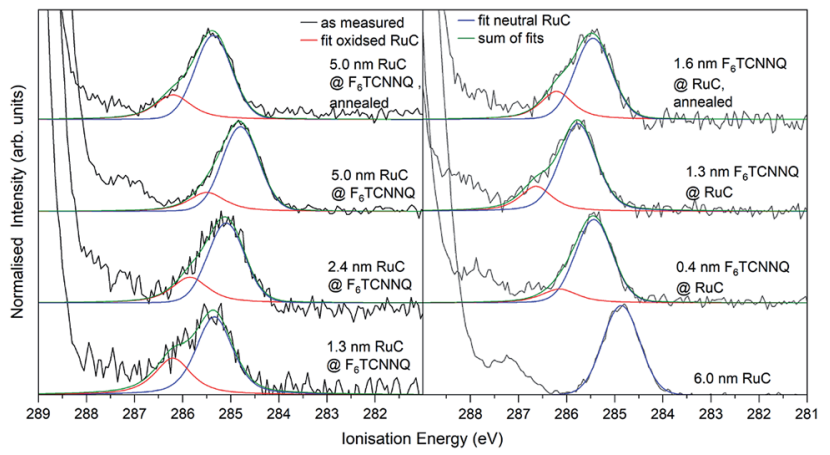

Fig. 7 Ru 3d core level XPS spectra of RuC $-F_{6} T C N N Q$ interfaces; left: $\mathrm{F}_{6} \mathrm{TCNNQ}$ as bottom layer with stepwise deposition of RuC; right: opposite sequence. The fit obtained from the neutral spectrum (bottom right) is shown in blue. All other spectra can be reconstructed by a superposition of the neutral spectrum and a second contribution which is assigned to oxidised molecules (red). Spectra were fitted with PseudoVoigt profiles with the software Fityk. ${ }^{25}$ 
molecules far below the interface. With higher coverages, the spectrum is increasingly dominated by oxidised RuC molecules close to the interface, because electrons from deeper lying molecules cannot escape the sample anymore. Analysis of the $\mathrm{S}$ $2 \mathrm{p}$ and $\mathrm{P} 2 \mathrm{p}$ spectra revealed no change in peak shape in the interface region with $\mathrm{F}_{6}$ TCNNQ. This indicates that predominantly the $\mathrm{Ru}$ centre of the RuC is oxidised while the ligand remains almost unaffected.

In order to confirm the charge transfer, the $\mathrm{N}$ 1s core level of $\mathrm{F}_{6}$ TCNNQ is analysed in Fig. 8. For pristine $\mathrm{F}_{6}$ TCNNQ (bottom curve on the left), the spectrum consists of a main feature at an $E_{\mathrm{i}}$ of $405.6 \mathrm{eV}$ and a satellite at $406.6 \mathrm{eV}$ stemming from a $\pi-\pi^{*}$ shake-up process. ${ }^{22}$ Since the Ru-complex does not contain nitrogen, the bottom curve on the right shows already the $0.3 \mathrm{~nm}$ coverage of $\mathrm{F}_{6}$ TCNNQ. The spectrum looks a lot different than the neutral spectrum with a large feature at $404.8 \mathrm{eV}$ and a smaller one at $402.7 \mathrm{eV}$. Since a film thickness of $0.3 \mathrm{~nm}$ constitutes the sub-monolayer regime (assuming flat lying molecules) it can be assumed that all molecules are reduced. Hence, it should be possible to reconstruct all other spectra by superimposing the neutral spectrum (blue curves) and the anion spectrum (red curves). This is indeed possible. For the sequence with $\mathrm{F}_{6}$ TCNNQ as bottom layer, the share of the anion spectrum to the total signal increases for growing film thicknesses. Analogous to the $\mathrm{Ru} 3 \mathrm{~d}$ spectra, this can be explained by the surface sensitivity of the method, shifting the 'information window' towards the interface region with increasing film thickness. For the opposite sequence, the share of the anion contribution decreases with higher coverages because molecules deposited on top of the first couple of layers are not reduced anymore. Again, annealing the sample drastically increases the amount of $\mathrm{F}_{6}$ TCNNQ molecules that find a 'partner' to interchange charge with, leading to a higher share of the anion spectrum to the overall $\mathrm{N}$ 1s signal for both deposition sequences.

Previous studies on $\mathrm{F}_{6}$ TCNNQ deposited on gold, ${ }^{23}$ graphene $^{24}$ and pentacene ${ }^{9}$ found that the $\mathrm{N} 1 \mathrm{~s}$ anion spectrum was shifted in binding energy but did not differ significantly in peak shape from the neutral spectrum. In the present case the $\mathrm{N} 1 \mathrm{~s}$

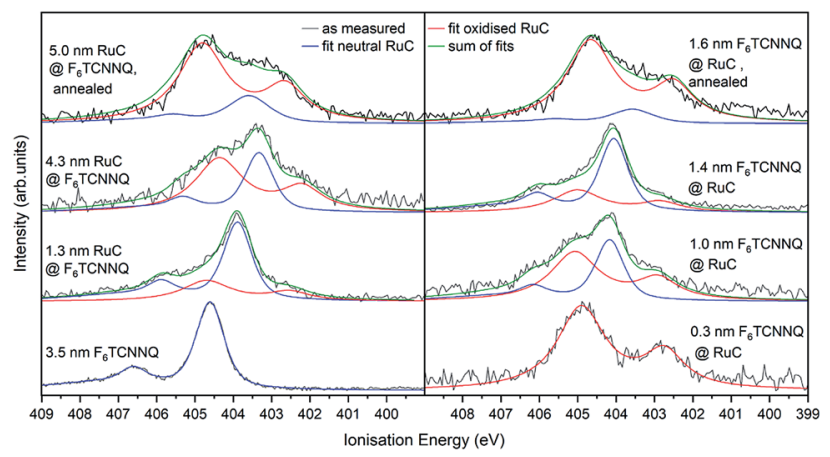

Fig. $8 \mathrm{~N}$ 1s XPS spectra of RuC-F $F_{6} T C N N Q$ interfaces; left: $F_{6} T C N N Q$ as bottom layer with stepwise deposition of RuC; right: opposite sequence. The neutral spectrum (bottom right) and the reduced spectrum (bottom left) were fitted by PseudoVoigt profiles (blue and red) curves. All other spectra could be reconstructed by a superposition of those two contributions. anion spectrum indeed differs strongly in peak shape. This indicates that in our case the nitrogen atoms are not equivalent anymore due to an asymmetric charge distribution on the molecule. This indicates a reaction of one of the amino groups of $\mathrm{F}_{6}$ TCNNQ with the RuC. This result is in accordance with the optical absorption measurements (Fig. 6) which also indicate that the charge transfer is not purely ionic.

Finally, the UP spectrum of the valence region is shown in Fig. 9 with the work function marked with vertical lines and again stacked in dependence of film coverage. The valence spectrum of pristine $\mathrm{F}_{6}$ TCNNQ is shown on the left (black curve). The high intensity feature with an onset at around $8.0 \mathrm{eV}$ can be attributed to the highest occupied molecular orbital (HOMO) of the molecule. The detected intensity at lower $E_{\mathrm{i}}$ 's can be attributed to the gold substrate. Moreover, since the work function of gold (around $5 \mathrm{eV}$ ) is smaller than the electron affinity of $\mathrm{F}_{6}$ TCNNQ (5.6 eV (ref. 17)), a charge transfer from the substrate to $\mathrm{F}_{6}$ TCNNQ is possible. In this case, the intensity could be assigned to the filled former LUMO of the molecules in the interface region to the substrate. ${ }^{23}$

With increasing coverage of RuC, the previously discussed valence structure of the RuC appears. It is not possible to clearly identify the LUMO occupation of $\mathrm{F}_{6}$ TCNNQ, since it is overlapping with the HOMO of the RuC. The reverse sequence shows the pristine RuC spectrum as bottom layer (black curve). With a low coverage of $0.4 \mathrm{~nm}$ of $\mathrm{F}_{6}$ TCNNQ, the two low $E_{\mathrm{I}}$ features are not clearly distinguishable anymore, partly due to the attenuation of the RuC signal by $\mathrm{F}_{6}$ TCNNQ and also the depletion of the RuC's HOMO and the filling of the overlapping $\mathrm{F}_{6}$ TCNNQ LUMO. The two topmost violet curves show the spectra after tempering. Both spectra look similar with a broad feature with an onset of $5.5 \mathrm{eV}$. This is in consistence with the XPS results and shows intermixing of the molecules independent from the deposition sequence.

The work function (vertical lines in Fig. 9) increases by about $1 \mathrm{eV}$ when depositing $0.4 \mathrm{~nm}$ of $\mathrm{F}_{6}$ TCNNQ (Fig. 9 - right) while it

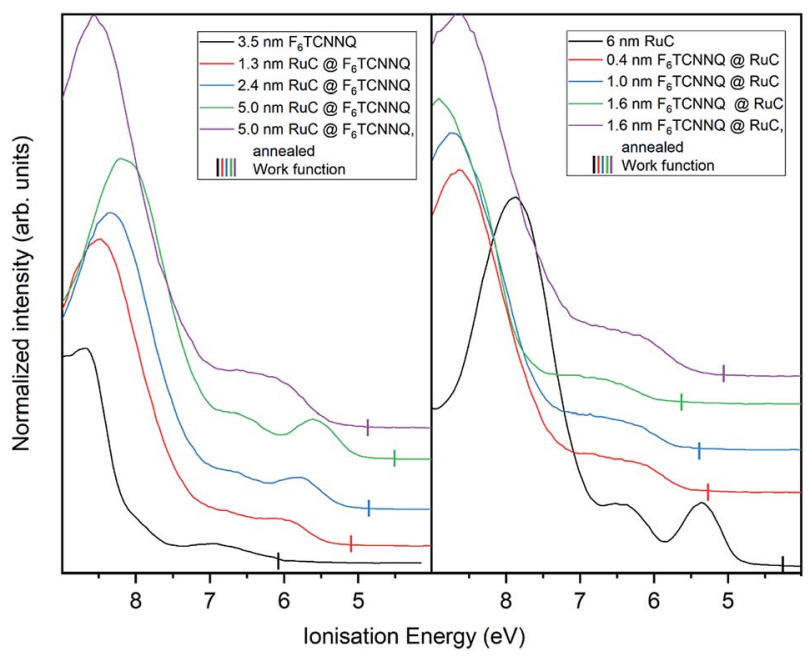

Fig. 9 UP spectra of valence region of RuC $-\mathrm{F}_{6} T C N N Q$ interfaces; left: $\mathrm{F}_{6} \mathrm{TCNNQ}$ as bottom layer with stepwise deposition of RuC; right: opposite sequence. The measured work functions for each deposition step are indicated by vertical lines. 
decreases by roughly the same amount for the opposite deposition sequence (Fig. 9 - left). This is further indication of a charge transfer since it reflects an immediate shift of the Fermi level when the RuC is in contact with $\mathrm{F}_{6}$ TCNNQ. Moreover, no occupied states close to the Fermi level (work function) are detected which indicates that the transferred charges are localised on their respective molecules.

\section{Conclusion}

In summary, we have synthesised an archetype ruthenium complex trans-[Ru(dppe $\left.)_{2}(\mathrm{~T})_{2}\right]$ and determined its fundamental electronic and optical properties. Optical absorption spectroscopy on spin-coated thin films revealed an optical gap of $3.1 \mathrm{eV}$ while an ionisation potential of about $5.0 \mathrm{eV}$ was determined by photoemission spectroscopy on vacuum evaporated films. Electrochemical analysis yielded similar values for the molecule in solution. A charge transfer reaction with the strong electron acceptor $\mathrm{F}_{6}$ TCNNQ was probed by optical absorption in a dissolved mixture of both materials. Furthermore, a charge transfer interface was formed by thermal evaporation and analysed by photoemission spectroscopy. Oxidised trans $-\left[\mathrm{Ru}(\mathrm{dppe})_{2}(\mathrm{~T})_{2}\right]$ and reduced $\mathrm{F}_{6}$ TCNNQ molecules were identified at the interface. The core level XPS spectra revealed that oxidation of trans$\left[\mathrm{Ru}(\mathrm{dppe})_{2}(\mathrm{~T})_{2}\right]$ occurs predominantly at the $\mathrm{Ru}$ centre while strong changes in the $\mathrm{N} 1 \mathrm{~s}$ spectra of the reduced acceptor show that electron and hole are clearly separated. However, the XPS and UV-vis aborption spectra differ from those previously reported for pure anionic $\mathrm{F}_{6}$ TCNNQ, which indicates that in the present case the charge transfer is not completely ionic.

\section{Conflicts of interest}

There are no conflicts to declare.

\section{Acknowledgements}

Financial support by the DFG (KN393/25, KN393/26) is gratefully acknowledged. We are grateful to Dr H. Komber for his support with NMR and thank R. Hübel, F. Thunig and M. Naumann for technical assistance. F. L. thanks the Fonds der Chemischen Industrie (FCI) for a Liebig Fellowship.

\section{Notes and references}

1 Y. Tanaka, Y. Kato, T. Tada, S. Fujii, M. Kiguchi and M. Akita, J. Am. Chem. Soc., 2018, 140, 10080-10084.

2 F. Schwarz, G. Kastlunger, F. Lissel, C. Egler-Lucas, S. N. Semenov, K. Venkatesan, H. Berke, R. Stadler and E. Lörtscher, Nat. Nanotechnol., 2016, 11, 170.

3 K. Sugimoto, H. Idei, Y. Tanaka and M. Akita, J. Organomet. Chem., 2017, 847, 121-131.
4 C.-Y. Wong, C.-M. Che, M. C. Chan, J. Han, K.-H. Leung, D. L. Phillips, K.-Y. Wong and N. Zhu, J. Am. Chem. Soc., 2005, 127, 13997-14007.

5 M.-Y. Choi, M. C.-W. Chan, S. Zhang, K.-K. Cheung, C.-M. Che and K.-Y. Wong, Organometallics, 1999, 18, 20742080.

6 P.-Y. Ho, H. Komber, K. Horatz, T. Tsuda, S. C. Mannsfeld, E. Dmitrieva, O. Blacque, U. Kraft, H. Sirringhaus and F. Lissel, Polym. Chem., 2020, 11, 472-479.

7 N. Gauthier, N. Tchouar, F. Justaud, G. Argouarch, M. P. Cifuentes, L. Toupet, D. Touchard, J.-F. Halet, S. Rigaut, M. G. Humphrey, et al., Organometallics, 2009, 28, 2253-2266.

8 D. Waas, F. Rückerl and M. Knupfer, Phys. Status Solidi B, 2018, 1800245.

9 M. Hantusch, R. Kuhrt and M. Knupfer, J. Phys. Chem. C, 2020, 124, 2961-2967.

10 R. Clark and J. Reid, Acta Crystallogr., Sect. A: Found. Crystallogr., 1995, 51, 887-897.

11 CrysAlis PRO, Rigaku Oxford Diffraction, Version 1.171.40.39a, 2019.

12 O. V. Dolomanov, L. J. Bourhis, R. J. Gildea, J. A. Howard and H. Puschmann, J. Appl. Crystallogr., 2009, 42, 339-341.

13 G. ShelXT, Acta Crystallogr., Sect. A: Found. Adv., 2015, 71, 38.

14 G. M. Sheldrick, Acta Crystallogr., Sect. C: Struct. Chem., 2015, 71, 3-8.

15 M. Seah and W. Dench, Surf. Interface Anal., 1979, 1, 2-11.

16 P. K. Koech, A. B. Padmaperuma, L. Wang, J. S. Swensen, E. Polikarpov, J. T. Darsell, J. E. Rainbolt and D. J. Gaspar, Chem. Mater., 2010, 22, 3926-3932.

17 H. Méndez, G. Heimel, S. Winkler, J. Frisch, A. Opitz, K. Sauer, B. Wegner, M. Oehzelt, C. Röthel, S. Duhm, et al., Nat. Commun., 2015, 6, 1-11.

18 P. Beyer, D. Pham, C. Peter, N. Koch, E. Meister, W. Brütting, L. Grubert, S. Hecht, D. Nabok, C. Cocchi, et al., Chem. Mater., 2019, 31, 1237-1249.

19 F. Zhang and A. Kahn, Adv. Funct. Mater., 2018, 28, 1703780. 20 V. Vijayakumar, P. Durand, H. Zeng, V. Untilova, L. Herrmann, P. Algayer, N. Leclerc and M. Brinkmann, J. Mater. Chem. C, 2020, DOI: 10.1039/D0TC02828B.

21 J. Chastain and R. C. King Jr, Handbook of X-ray photoelectron spectroscopy, Perkin-Elmer Corporation, 1992, vol. 40, p. 221.

22 J. M. Lindquist and J. C. Hemminger, J. Phys. Chem., 1988, 92, 1394-1396.

23 R. Kuhrt, M. Hantusch, M. Knupfer and B. Büchner, Surf. Interface Anal., 2020, 52, 953-956.

24 C. Christodoulou, A. Giannakopoulos, G. Ligorio, M. Oehzelt, M. Timpel, J. Niederhausen, L. Pasquali, A. Giglia, K. Parvez, K. Müllen, et al., ACS Appl. Mater. Interfaces, 2015, 7, 19134-19144.

25 M. Wojdyr, J. Appl. Crystallogr., 2010, 43, 1126-1128. 